\title{
Effects of Instruction of English Learning Design Focusing on Individual Learners' Needs
}

\author{
Mayumi Abe* and Chiharu Kogo**
}

(The original Japanese article was published in Japan Journal of Educational Technology, Vol. 43, No. 3, 2019, pp. 231-238.)

\begin{abstract}
In self-directed language learning, it may be more effective for learners to design and carry out a learning plan that matches their individual needs. This study was conducted in a course at a Japanese university to examine the effects of instruction concerning English language learning design on learners' planning and learning performance. The results showed that: (1) instruction in learning design helped learners create learning plans that matched their needs, and (2) learning plans matching learners' preferences increased their motivation to learn, while plans matching learners' preferences and learning environment improved the completion rate of the plans. The results suggest the importance of providing instruction in learning design, focusing on individual learners' needs, especially their preferences.
\end{abstract}

Keywords language education, self-directed learning, personalized learning, individual needs, learning design

\section{Introduction}

Learner-centered personalized learning is gaining popularity, both inside and outside the classroom, over the traditional practice of group instruction ${ }^{[1]}$. The greatest benefit of personalized learning is that it provides a learning experience that matches the needs and pace of individual learners, which is difficult to achieve in group instruction. To provide such an experience, Bray and McClaskey ${ }^{[1]}$, for example, proposed the creation of a personal learner profile to help understand the strengths, weaknesses, and needs of each learner, as well as the selection of a learning approach that suits individual characteristics.

In the field of language education, it has been widely recognized that learning inside the class is not sufficient on its own, and that independent efforts outside of class are also necessary for language acquisition $^{[2]}$. Many studies focusing on the individual attributes of learners in relation to second-language acquisition have also been conducted ${ }^{[3]}$. However, few studies have investigated how the learning content of self-directed learning outside of class is selected, and whether such selection matches the individual needs of the learner ${ }^{[2]}$.

\footnotetext{
* Graduate School of Human Sciences, Waseda University, Japan

** Faculty of Human Sciences, Waseda University, Japan
}

Needs analysis in the field of language education in relation to research and practice has certainly been undertaken, and the importance of needs analysis as part of curriculum design has been recognized ${ }^{[4],[5]}$. Needs analysis is the cornerstone of program design, especially in English for specific purposes (ESP) education, with targeted research being conducted in that field ${ }^{[6], ~[7]}$. However, the focus of needs analysis, even in those studies, continues to be on group instruction rather than on personalized learning ${ }^{[7]}$.

These research trends suggest that group education provided by schools and private institutions has been the predominant area of interest in English language learning. Even though independent learning outside of class has been recognized as valuable, it may have been regarded as peripheral to learning. However, many English learners are not part of group-learning environments. Moreover, out-of-class learning is important, even for learners enrolled in schools. Given this context, it is likely that conducting trials and research in relation to learning designs that include a needs analysis will help improve the quality of self-directed learning outside of class.

Consequently, this study was conducted to explore how self-directed learning among English language learners may be improved. Learning design instruction was provided in a course at a Japanese university, and this study aimed to test the following two hypotheses:

(1) Instruction concerning learning design helps learners 
create learning plans that match their needs.

(2) The degree of agreement between learning plans and individual needs affects learners' motivation and the completion rate of the plans.

In this study, "learning design" refers to a comprehensive process, ranging from needs analysis and goal setting to creating a learning plan and reflection. These hypotheses were tested through providing instruction on learning design over a period of one semester in the university course.

\section{Methods}

\subsection{Target and Duration of the Study}

The study was conducted in a course with the theme of self-directed learning in English at X University. The participants of the study were from two classes comprising 43 first-year students (17 males, 26 females) at the university. The course was held between April and July 2018. It consisted of a total of 14 classes, involving one 90-min class per week.

\subsection{Course Outline and Assignments}

The following content was taught in the course with the aim of helping learners acquire the knowledge and skills necessary for learning design (Table 1).

Class 1, "Introduction," explained the significance of independent learning outside the classroom as well as the importance of the skills required to design one's own learning. Classes 2 to 5 provided instructions on learning strategies for each skill in relation to key areas, such as vocabulary, reading, and listening, to help improve language ability. In addition to introducing learning resources, activities were conducted that encouraged the learners to think about their individual level in connection with each skill and genre of English used in the learning resources. In Class 7, "Needs Analysis \& Goal Setting," each student made full use of what they had learned up to that point concerning each language skill to conduct their own individual needs analysis and goal setting. In Class 8, "Designing Learning Plan 1," the learners designed concrete learning plans. For this, the learners were instructed to consider, based on the needs analysis and goal setting conducted in Class 7, what they needed to learn (resource), how they would learn it (strategy), and when and where they would learn it. Classes 10 to 12 dealt with topics related to learning management. In Class 10, "Motivation," the learners participated in activities to help them relate basic motivation concepts, such as intrinsic motivation, extrinsic motivation, and self-efficacy, to their own learning. In Class 11, "Time

Table 1. Outline of classes and topics

\begin{tabular}{|c|c|c|c|}
\hline Class & Topic & Assignment & Vocabulary Size Test \\
\hline 1 & Introduction & Intro Questionnaire & \\
\hline 2 & Learning Strategies \& Resources: Vocabulary & & pre-test \\
\hline 3 & Learning Strategies \& Resources: Reading & LP0 Learning Plan & \\
\hline 4 & Learning Strategies \& Resources: Listening & & \\
\hline 5 & Learning Strategies \& Resources: Speaking \& Writing & LP0 Results Report & \\
\hline 6 & In-class Test (no instruction) & & \\
\hline 7 & Needs Analysis \& Goal Setting & & \\
\hline 8 & Designing Learning Plan 1 & LP1 Learning Plan & \\
\hline 9 & Sharing Learning Plan 1 & & \\
\hline 10 & Motivation & & \\
\hline 11 & Time Management & & \\
\hline 12 & PDCA Cycle in Self-Directed Learning & LP1 Results Report & \\
\hline 13 & Designing Learning Plan 2 & LP2 Learning Plan & post-test \\
\hline 14 & Poster Presentations & & \\
\hline
\end{tabular}

$\mathrm{LP}=$ Learning Plan 
Management," the learners prepared daily or weekly schedules and engaged in activities to review their time management. In Class 12, "Plan-Do-Check-Act (PDCA) Cycle in Self-Directed Learning," the learners obtained an overview of the flow of self-directed learning through learning about the processes of planning, doing, checking, and acting in relation to their learning plans.

Throughout the semester, the students submitted the assignments they had created using the Moodle online learning management system. Some of the assignments were selected for analysis in this study. The medium of instruction in the classes and for the assignments was English, as per the university's policy.

In the first class, a questionnaire survey was conducted in which the students carried out a self-analysis of their independent learning of English to that point (Intro Questionnaire). Additionally, three learning plans were designed during the semester. Reports on the results were submitted after completing the first learning plan (LP0) and the second learning plan (LP1). The completion periods for the learning plans were approximately two weeks for LP0 and four weeks for LP1. The result reports after the completion of the third learning plan (LP2) could not be collected because it was a learning plan intended for the summer holidays. The learning content to be included in the learning plans was determined by the students themselves, and the learning resources, strategies, and other relevant components were selected according to the needs of each learner. However, the learners were required to include one item related to vocabulary learning in their plans. Additionally, the students took online vocabulary size tests $^{[8]}$ twice during the semester to assess their vocabulary levels. The results of the vocabulary size tests were used for the pre- and post-test analyses in this study.

\subsection{Contents of the Surveys}

To investigate whether the students were able to create a learning plan that matched their individual needs as an effect of instruction in learning design, surveys were conducted to examine the degree of agreement between the learning plans and the individual needs of the student. The surveys were conducted four times in relation to the Intro Questionnaire, LP0, LP1, and LP2. Moreover, surveys on motivation and the completion rate were included as part of the result reports for LP0 and LP1 to assess how motivation and the completion rate were affected by the degree of agreement between the learning plans and the needs of the students.

Following Brown ${ }^{[6]}$ (pp. 19-20), the five criteria listed below were used to assess the degree of agreement between the learning plans and the needs of individual learners:

(1) Level: Current level of the learner (hereinafter referred to as level),

(2) Skill: Skill that the learner needs to develop to meet the objective (hereinafter referred to as skill),

(3) Genre: Genre that the learner intends to study (hereinafter referred to as genre),

(4) Preference: Preference for the learner (hereinafter referred to as preference), and

(5) Environment: Learning environment of the learner (hereinafter referred to as environment).

The Intro Questionnaire and surveys for each of the three learning plans (LP0 to LP2) consisted of a 5-point Likert rating scale, with answers ranging from: 1. "Does not fit my needs at all"; 2. "Does not fit my needs so much"; 3. "Neutral"; 4. "Somewhat fits my needs"; and 5. "Fits my needs." The students used this rating scale in the Intro Questionnaire to self-check the independent learning they were doing at that point. The surveys included in the three learning plans were used to self-check the learning content of each plan. Before conducting the Intro Questionnaire survey, the students were given both a verbal explanation and a handout containing concrete examples of the five items in relation to indicating the degree of agreement with the learners' needs. Additionally, since this was the first class, the survey questions and the five options were written in both English and Japanese in consideration of the students' level of understanding of English. The survey questions and the five options in the questionnaires for LP0 and LP1 were written in English only because the students were expected to have understood them at that time. Moreover, the five criteria used to assess the degree of agreement with their needs were presented to the students before they created their learning plans, and the students were advised to create learning plans that matched their needs as closely as possible. However, despite the instructions, it may have been difficult for the students to apply their needs directly to the learning plans. Therefore, the self-checks in LP0, LP1, and LP2 encouraged them to review what they had included in their learning plans and were included in our study as an 
index to measure the effects of instruction.

Motivation and the completion rate were entered for each item of learning content as part of the reflection in the result reports created by the students after completing LP0 and LP1.

The following four questions, based on the ARCS model (attention, relevance, confidence, and satisfaction), were included in the survey to evaluate motivation. After explaining the questions verbally, the students were instructed to provide their answers using a 5-point Likert scale ranging from 1 indicating "No" to 5 indicating "Yes" (self-evaluation based on ARCS, hereinafter referred to as "ARCS evaluation"):

(1) Were you interested in the learning activity? (attention),

(2) Do you think learning was effective in improving your skill? (relevance),

(3) Were you able to carry out the activity with confidence? (confidence), and

(4) Are you satisfied with your performance and achievement? (satisfaction).

The questions were presented only in English because the words and phrases contained in them were used repeatedly in the class, and the target students were expected to understand them when the questions were first shown to the students in Class 5. The questions were also verbally explained to the students.

The students were asked to evaluate the completion rate of the learning plans using percentages. The amount, time, and frequency of learning decided by the students in their learning plans were used to assess the completion rate.

The students were informed beforehand that their answers in relation to the degree of agreement with the learning plan, ARCS evaluation, and the completion rate would not be included in their grades. Verbal and written information was also provided to the students that the submitted assignments might be used for research purposes in a non-personally identifiable manner, and that participation was voluntary and would not affect their grades. Consent was obtained from all students who participated in this study.

\section{Results}

\subsection{Number of Valid Responses}

Of the 43 students who participated in this study, 27 (5 males, 22 females) submitted the Intro Questionnaire and all the learning plans (LP0, LP1, and LP2) with no incomplete responses. These 27 students also reported pre- and post-test results of the vocabulary size test. Consequently, only these responses were considered valid and were used in the analyses to compare the four learning plans and the pre- and post-vocabulary size tests. Thirty-nine valid responses were received for LP0 (90.7\% response rate), 38 for LP1 (88.4\% response rate), and 29 for LP2 (69.8\% response rate). The process and timing for collecting LP2 did not proceed as expected, which led to a significant drop in its potential response rate.

The responses considered valid and used for assessing the learning plans and for undertaking result report analyses comprised those from respondents who submitted both learning plans and result reports for LP0 and LP1.

Thirty-nine respondents submitted both LP0 and related result reports ( 13 males, 26 females). Of the 86 learning content items in the learning plans created by these respondents, 75 had no incomplete responses for the degree of agreement with the learning plan, ARCS evaluation, and the completion rate. Consequently, these 75 items were used for the LP0 and result report analyses. The number of learning content items included in the learning plans was one to two per person.

Thirty-eight respondents submitted LP1 and related result reports (13 males, 25 females). Of the 126 learning content items in the learning plans created by these respondents, 93 had no incomplete responses for the degree of agreement with the learning plan, ARCS evaluation, and the completion rate. Consequently, these 93 items were used for the LP1 and result report analysis. The number of learning content items included in the learning plans was one to three per person.

Since the number of items that could be included in the learning plans as learning content was unlimited, some respondents included more than one learning content item for LP0 and LP1. However, there was no significant bias in the number of items identified by each respondent, and the responses concerning the degree of agreement with the learning plan, ARCS evaluation, and the completion rate varied according to the learning content, even for the same learner. Therefore, all items were assigned the same weight in the analyses. 
Table 2. Descriptive statistics and effect sizes of degree of agreement in needs

$(n=27)$

\begin{tabular}{|c|c|c|c|c|c|c|c|}
\hline & \multicolumn{4}{|c|}{ Mean $(S D)$} & \multicolumn{3}{|c|}{ Effect size $(d)$} \\
\hline & Intro & LP0 & LP1 & LP2 & Intro-LP0 & LP0-LP1 & LP1-LP2 \\
\hline \multicolumn{8}{|l|}{ Learning Plan } \\
\hline All needs & $3.7(0.43)$ & $4.2(0.42)$ & $4.3(0.37)$ & $4.3(0.73)$ & $\underline{1.02}$ & 0.29 & 0.07 \\
\hline Level & $3.8(0.77)$ & $4.2(0.56)$ & $4.2(0.54)$ & $4.2(0.81)$ & $\underline{0.58}$ & 0.01 & 0.03 \\
\hline Skill & $3.6(0.53)$ & $4.2(0.55)$ & $4.4(0.51)$ & $4.3(0.77)$ & $\underline{1.06}$ & 0.49 & 0.17 \\
\hline Genre & $3.7(0.78)$ & $4.1(0.65)$ & $4.4(0.54)$ & $4.3(0.80)$ & $\underline{0.62}$ & 0.45 & 0.05 \\
\hline Preference & $3.8(0.69)$ & $4.0(0.78)$ & $4.2(0.60)$ & $4.3(0.85)$ & 0.25 & 0.28 & 0.11 \\
\hline Environment & $3.8(0.69)$ & $4.3(0.78)$ & $4.1(0.91)$ & $4.4(0.85)$ & $\underline{0.63}$ & 0.18 & 0.32 \\
\hline
\end{tabular}

*Figures in boldface are above the small standard; underlined figures are above the medium standard.

\subsection{Changes in the Degree of Agreement with Needs in the Learning Plans}

To verify the effect of instruction on learning design, paired $t$-tests for each of the five needs criteria were performed between the Intro Questionnaire and LP0, LP0 and LP1, and LP1 and LP2, using the mean value of all the items listed under needs. Furthermore, the effect size $d$ was calculated for each pair to study the degree of change (Table 2, Fig. 1). Cohen's ${ }^{[9]} d$ was used as the reference to set the magnitude of the effect size, with $d=0.20$, small; $d=0.50$, medium; and $d=0.80$, large.

Between the Intro Questionnaire and LP0, there was a significant difference in the average of all items under needs $(p<.001)$. When comparing each item, there were significant changes in level $(p<.05)$, skill $(p<.001)$, genre $(p<.05)$, and environment $(p<.01)$. The scores for LP0 were higher than those for the Intro Questionnaire.

Between LP0 and LP1, there was no significant change at the $5 \%$ level in the average of all items under needs. However, a small effect size was observed $(d=0.29)$. When comparing each item, there was a significant change in genre $(p<.05)$, and skill was marginally significant $(p<.10)$. All scores were higher for LP1.

Between LP1 and LP2, there was no significant change at the $5 \%$ level in the average of all items under needs, and there was almost no effect size. No significant differences were found in the comparisons between the items.

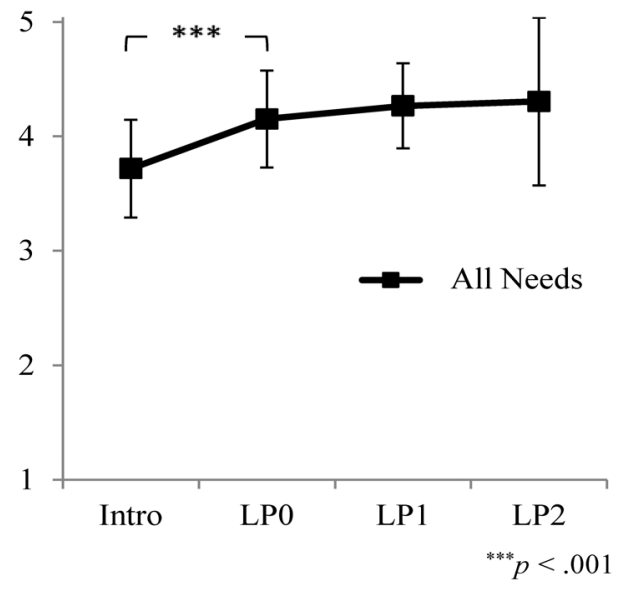

Figure 1. Changes in the degree of agreement with the five needs criteria

\subsection{Pre- and Post-Test Comparisons of Vocabulary Size}

The results of the vocabulary size tests conducted at the beginning and end of the semester were compared as pre- and post-test results. The average pre-test vocabulary size of the students was 7007.41 words $(S D=1104.86)$, and the average post-test vocabulary size was 7277.78 words $(S D=1200.75)$. A paired $t$-test showed no significant difference, while the effect size was small $(d=0.23)$. 
Table 3. Descriptive statistics of learning contents in LP0 and LP1

\begin{tabular}{lcc}
\hline & \multicolumn{2}{c}{ Mean $(S D)$} \\
\cline { 2 - 3 } & LP0 $(n=75)$ & LP1 $(n=93)$ \\
\hline Learning Plan & & \\
All Needs & $4.1(0.56)$ & $4.2(0.48)$ \\
Level & $4.1(0.89)$ & $4.1(0.77)$ \\
Skill & $4.1(0.72)$ & $4.3(0.69)$ \\
Genre & $4.1(0.86)$ & $4.3(0.70)$ \\
Preference & $4.1(0.93)$ & $4.2(0.77)$ \\
Environment & $4.3(0.87)$ & $4.2(1.00)$ \\
\hline Results Report & & \\
ARCS Evaluation & $3.4(0.78)$ & $3.7(0.86)$ \\
Completion Rate (\%) & $52.2(27.05)$ & $61.7(23.64)$ \\
\hline
\end{tabular}

\subsection{Effect of the Degree of Agreement with Needs on Motivation and Completion Rate}

To investigate the effects of the degree of agreement with needs on motivation and the completion rate, we analyzed LP0 and LP1 for which the respondents had submitted both learning plans and result reports. The mean values of the learning content items were used in the analyses. The respective mean values and standard deviations of LP0 and LP1 are listed in Table 3.

\subsubsection{Analysis of LPO}

A multiple stepwise regression analysis was performed to study the effect of each of the five criteria on the ARCS evaluation. Of the five criteria, the standard partial regression coefficient for preference was found to be significant $\left(\beta(75)=.29, R^{2}=.08, p<.05\right)$.

A multiple stepwise regression was also performed to study the effect of each of the five criteria on the completion rate. Of the five criteria, the standard partial regression coefficient for the environment was found to be significant $\left(\beta(75)=.24, R^{2}=.06, p<.05\right)$ (Fig. 2).

\subsubsection{Analysis of LP1}

A multiple stepwise regression analysis was performed to study the effect of each of the five criteria on the ARCS evaluation. Of the five criteria, the standard partial regression coefficient for preference was found to be significant $\left(\beta(93)=.43, R^{2}=.19, p<.001\right)$.

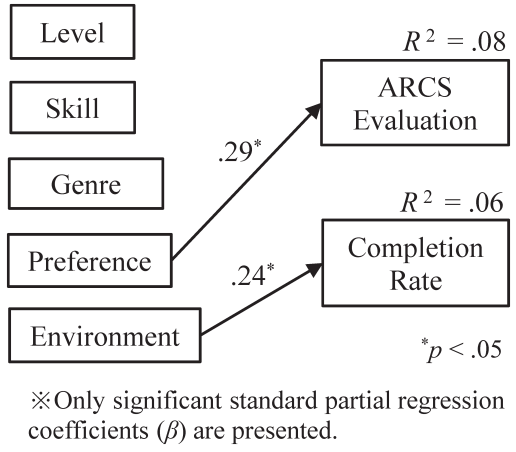

Figure 2. Multiple regression analysis for LP0

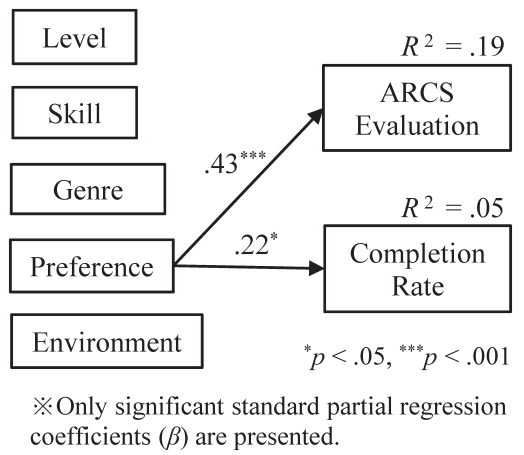

Figure 3. Multiple regression analysis for LP1

A multiple stepwise regression analysis was also performed to study the effect of each of the five criteria on the completion rate. Of the five criteria, the standard partial regression coefficient for preference was found to be significant $\left(\beta(93)=.22, R^{2}=.05, p<.05\right)$ (Fig. 3).

\section{Discussion}

\subsection{Effects of Instruction on Learning Design}

In terms of the first hypothesis of this study, "Instruction concerning learning design helps learners to create learning plans that match their needs," the following results were obtained.

The degree of agreement with the needs criteria in LP0 was higher than that in the Intro Questionnaire, with the $t$-test showing a significant difference. Moreover, after analyzing each item, significant dif- 
ferences were found in level, skill, genre, and environment. Instruction on vocabulary learning and reading strategy was provided after completion of the Intro Questionnaire and before LP0, and the students created learning plans for reading and vocabulary in LP0. The difference in the degree of agreement with needs at this point can be attributed to the fact that instruction on strategy improved the students' metacognitive consciousness of learning design, and they were able to make choices that better matched their individual needs. Level, skill, and genre were also included in the instruction of learning design, and the higher scores for these criteria would also appear explicable as resulting from the effects of instruction.

Learning strategies for listening, speaking, and writing were provided after completing LP0 and before LP1. Additionally, instruction on self-needs analysis and goal setting was provided before creating LP1. The degree of agreement with needs was higher again in LP1 than in LP0, but with no significant difference. Three possible reasons may explain this finding. First, instruction in learning design is likely to have a strong effect when first introduced but repeating it several times does not necessarily improve the results proportionately. Second, the effect of instruction on self-needs analysis and goal setting was simply not as significant as expected. Third, the average score in LP0 was 4.1 out of 5, which created a ceiling effect for scores in LP1.

Instruction for maintaining motivation, schedule management, and the PDCA cycle was provided after completing LP1 and before LP2. However, there were no significant differences in the degree of agreement with needs. This finding could be due to a weakening in the effect of instruction and the ceiling effect of the scores, as previously noted, in relation to LP0 and LP1. However, although there were no significant differences between LP0 and LP1, and between LP1 and LP2, the effect size for LP0 and LP1 was small, while there was almost no effect size for LP1 and LP2. From these findings, it would appear that the degree of agreement with needs gradually settled at a fixed level after an initial rise. Furthermore, the fact that the scores did not fall when moving from LP0 to LP1 and then to LP2 would appear to be because of the continuing effects of instruction. Considering these possibilities, the effects of instruction, including its frequency and duration, need to be further investigated.

All students who participated in this study were instructed to include vocabulary learning in their plans. Consequently, pre- and post-test evaluations of vocabulary size were conducted as one means to measure the effects of instruction. No significant difference was observed between the pre- and post-test results. However, since a small effect size was observed, it is possible that instruction in learning design, planning by the students, and execution of the plans led to an improvement in vocabulary.

\subsection{Effects of Individual Learning Plans on Motivation and the Completion Rate}

In terms of the second hypothesis of this study, "The degree of agreement between learning plans and individual needs will affect learners' motivation and the completion rate of the plans," the following results were obtained.

First, the multiple regression analysis between the degree of agreement with needs and ARCS evaluation showed that, of the five criteria, preference alone had an effect on ARCS evaluation in both LP0 and LP1. This finding suggests that, regarding the degree of agreement with needs in the learning plan, motivation for learning increases if the learning content matches needs such as learners' interests and likes. Motivation in relation to learning content affects future learning efforts. Therefore, it is important to develop learning plans that increase motivation.

Second, the multiple regression analysis between the degree of agreement with needs and the completion rate showed that, of the five criteria, the effect of environment in LP0 and preference in LP1 on the completion rate was significant. In other words, the possibility of successfully undertaking a learning plan was higher when the learning plan suited the learners' learning environment and preferences. The construction of a good learning plan is of no use if it is not executed. Therefore, when designing a learning plan, it is important to consider the learning environment and preferences to increase the probability of learners following through with the learning plan.

From these results, two guidelines can be deduced to support self-directed learners of English. First, when teaching learning design, it is recommended to teach learners to select learning resources and strategies that match their individual preferences and learning envi- 
ronments. While this study provides further evidence supporting the efficacy of instruction in learning design generally, additional support can be given through helping learners create a learning plan that increases motivation and is easy to follow through encouraging them to pay attention to factors such as their preferences and learning environment. Second, it is recommended that learning materials that match learners' preferences and learning environments be provided. Although these features can be expected to vary depending on the learner, it is possible to match the preferences and learning environments of many learners through providing learning content that catches their interest, a learning system that stimulates learning, and learning media that are easy to use anytime and anywhere.

Since the participants of this study were all university students, it was possible to provide in-class instruction on learning design with an instructor. However, learners who study independently and who are not part of a group learning environment may find it difficult to receive such direct instruction. Moreover, it can be assumed that learners have diverse views about learning and different purposes for pursuing it. There is scope for assessing how the results of this study could be used to provide support for even more effective learning design that would cover a wider range of learners in the future.

Furthermore, while improving motivation and the completion rate are important, learning effectiveness should not be ignored when teaching learning design. A plan that considers the current level of learners, their target genre, and the skills that need to be developed to achieve their goals is a significant factor in ensuring learning effectiveness. Future research is needed to determine how learning plans can be created that achieve high learning effectiveness while improving motivation and the completion rate.

\section{Conclusion}

In this study, instruction in learning design was provided in a course at a university to explore the hypothesis that creating a learning plan that matches the individual needs of learners would be effective in improving the quality of self-directed learning of English. The study investigated: (1) the effects of instruction on learners' planning; and (2) the effects of the degree of agreement between planning and needs on subsequent learning. The results showed that learners were able to create learning plans that better matched their individual needs after receiving instruction on learning design. Moreover, the results indicated that, in relation to individual needs, plans that matched learners' preferences increased their motivation for learning, while plans that matched learners' preferences and learning environment improved the completion rate of the plans. The results suggest the importance of providing instruction in learning design that meets the individual needs of learners, especially that their preferences be included in planning, to ensure more effective self-directed learning of English.

\section{Author Notes}

This study is a reanalysis and expansion of previous studies presented by Abe and $\mathrm{Kogo}^{[10],[11]}$.

\section{Acknowledgments}

We would like to express our sincere gratitude to everyone involved in this research for their support and cooperation.

\section{References}

[1] B. Bray and K. McClaskey, Make Learning Personal: The What, Who, WOW, Where, and Why. Thousand Oaks, CA, USA: Corwin, 2015.

[2] P. Benson, Teaching and Researching Autonomy, 2nd ed. London, UK: Longman, 2011.

[3] T. Takeuchi, "What we have learned from language learner research: From an individualistic perspective to a holistic one," in Learner Development in English Education: Learner Factors and Autonomous Learning, H. Kojima, N. Ozeki, and T. Hiromori, Eds., Tokyo, Japan: Taishukan-shoten (in Japanese), 2010, ch. 1, pp. 3-20.

[4] J. D. Brown, The Elements of Language Curriculum: A Systematic Approach to Program Development. Boston, MA, USA: Heinle Cengage Learning, 1995.

[5] I. S. P. Nation and J. Macallster, Language Curriculum Design. New York, NY, USA: Routledge, 2010.

[6] J. D. Brown, Needs Analysis and English for Specific Purposes. New York, NY, USA: Routledge, 2016.

[7] C. Iwai, Needs Analysis of ESP Education: Aiming for Global Human Resource Development in Industry and Academia. Osaka, Japan: Osaka Municipal Universities Press (in Japanese), 2014. 
[8] I. S. P. Nation, "Vocabulary Size Test." VocabularySize.com. https://my.vocabularysize.com/ (accessed Jan. 4, 2019).

[9] J. Cohen, “A power primer," Psychol. Bull., vol. 112, no. 1, pp. 155-159, Jul. 1992.

[10] M. Abe and C. Kogo, "Effects of needs-matched planning on behavior and awareness of self-directed learners of English," (in Japanese), in Proc. 34th Annu. Conf. JSET, Sep. 2018, pp. 495-496.

[11] M. Abe and C. Kogo, "The effects of instruction of planning for self-directed learners of English," (in Japanese), Res. Rep. JSET Conf., vol. 18, no. 4, Oct. 2018, pp. 117-122.

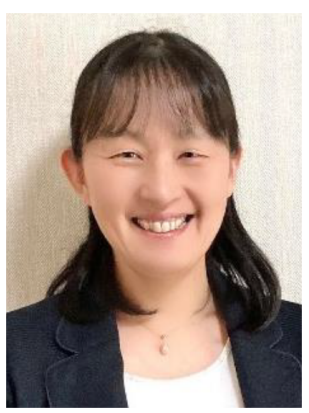

Mayumi Abe received her M.S.Ed. from Temple University and Ph.D. from Waseda University. She is currently an assistant professor at the Center for Higher Education Studies of Waseda University. Her research interests include instructional design, learning design, and second language education.

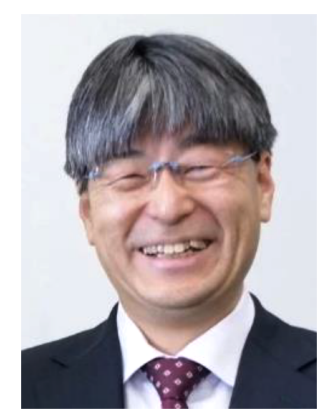

Chiharu Kogo received his B.A. and M.A. from Waseda University and his Ph.D. from Tokyo Gakugei University. He is a professor at the Faculty of Human Sciences at Waseda University. His research interests include educational technology, educational psychology, and Adlerian psychology. 